$\underline{\text { Original article }}$

\title{
Synovial deposition of wild-type transthyretin-derived amyloid in knee joint osteoarthritis patients
}

Tetsuo Takanashi ${ }^{1}$, Masayuki Matsuda ${ }^{3}$, Masahide Yazaki ${ }^{3}$, Hideshi Yamazaki ${ }^{1}$, Masashi Nawata ${ }^{2}$, Yoshiki Katagiri ${ }^{2}$, and Shu-ichi Ikeda ${ }^{3}$

1) Department of Rheumatology, Marunouchi Hospital, Matsumoto, Japan

2) Department of Orthopedic Surgery, Marunouchi Hospital, Matsumoto, Japan

3) Department of Medicine (Neurology and Rheumatology), Shinshu University School of Medicine, Matsumoto, Japan

Running title: Synovial deposition of wild-type TTR amyloid

Correspondence: Dr. Masayuki Matsuda, Department of Medicine (Neurology and Rheumatology), Shinshu University School of Medicine, 3-1-1 Asahi, Matsumoto 390-8621, Japan

Tel: +81-263-37-2673

Fax: +81-263-37-3427

e-mail: matsuma@shinshu-u.ac.jp 


\section{Abstract}

Objective: To investigate histological features of deposited amyloid in the synovial tissue and its clinical significance in knee joint osteoarthritis patients.

Methods: We prospectively enrolled 232 consecutive patients who underwent arthroplasty or total replacement of the knee joint for treatment of osteoarthritis. Congo red staining and immunohistochemistry were performed in the synovial tissue obtained at surgery. When transthyretin (TTR)-derived amyloid was positive, we analyzed all 4 exons of the TTR gene using the direct DNA sequencing method in order to detect mutations.

Results: We analyzed 322 specimens in this study. Twenty-six specimens $(8.1 \%)$ obtained from 21 patients (5 men and 16 women; mean, 79.0 44.6 years) showed deposition of amyloid, which was positively stained with the anti-TTR antibody. Eighteen patients showed inhomogeneous accumulations of amyloid in the loose connective tissue under the synovial epithelia sometimes with nodule formation, while in the remaining 3, small vessels in the adipose tissue were involved. Medical records of these patients revealed nothing remarkable in the clinical course, laboratory data or macroscopic intraarticular findings at surgery. No mutations were detectable in the TTR gene analysis.

Conclusion: Wild-type TTR-derived amyloid may affect the synovial tissue as a result of long-term mechanical stress or as a part of senile systemic amyloidosis in approximately $8 \%$ of knee joint osteoarthritis patients. No obvious clinical significance was found in synovial deposition of amyloid.

Keywords: knee joint, osteoarthritis, senile systemic amyloidosis, synovial tissue, wild-type transthyretin 
Abbreviations: $C R P=C$-reactive protein, $D A S 28=$ disease activity score including 28-joint count, ECG=electrocardiogram, MRI=magnetic resonance imaging, OA=osteoarthritis, $R A=$ rheumatoid arthritis, SSA=senile systemic amyloidosis, $T T R=$ transthyretin 


\section{Introduction}

Wild-type transthyretin (TTR) causes senile systemic amyloidosis (SSA) clinically characterized by slowly progressive heart failure due to cardiac involvement in elderly people [1-3]. According to several recent reports, however, this type of TTR-derived amyloid frequently affects soft tissues also, such as the tendons and ligaments, with no evidence of visceral organ involvement, resulting in carpal tunnel syndrome when the median nerve is compressed in the wrist joint $[4,5]$. Precise mechanisms by which such wild-type TTR-derived amyloid preferentially accumulates in the soft tissue remain unclear, but mechanical stress may play an important role in the pathogenesis [4]. Articular synovium is another soft tissue always exposed to mechanical stress. Hemorrhagic arthritis in the knee joint has been reported to occur as a complication of synovial TTR amyloid deposition in an aged savannah monkey [6]. In the present study we investigated whether or not synovial deposition of TTR amyloid is present in human specimens consecutively obtained at surgery from patients with osteoarthritis (OA), which causes degradation of articular cartilage and subchondral bone in conjunction with ageing. Synovial deposition of wild-type TTR-derived amyloid was seen in approximately $8 \%$ of the specimens, but its clinical significance remains unclear.

\section{Patients and Methods}

\section{$\underline{\text { Patients }}$}

From September 2010 to July 2011, 232 consecutive patients (31 men and 201 women; mean, 73.0 \pm 9.1 years), who underwent total replacement or arthroplasty of the knee joint in Marunouchi Hospital for treatment of OA were prospectively enrolled in this study. The diagnosis of OA was done according to the classification criteria proposed by the American Rheumatism Association [7]. We obtained the synovial tissue with a size of approximately $1 \mathrm{~cm}$ in diameter from these patients at surgery. The 
specimens were fixed with $20 \%$ formalin neutral buffer solution (Wako Pure Chemical, Osaka, Japan), and processed for histological examinations. Clinical information on these patients, such as macroscopic intraarticular findings at surgery, associated diseases, acute phase reactants and abnormalities on electrocardiogram (ECG) and chest X-ray, was also obtained from their medical records. This study was approved by the Ethical Committee of Marunouchi Hospital (No. 10-01), and written informed consent was obtained from each patient. We excluded patients with the following amyloidogenic conditions: chronic inflammatory diseases, particularly active rheumatoid arthritis (RA) at surgery, multiple myeloma, $\mathrm{M}$ protein in the serum and/or urine, chronic renal failure under hemodialysis and previously diagnosed systemic amyloidosis, including familial amyloid polyneuropathy and SSA. Active RA was defined as a state showing a disease activity score including a 28-joint count (DAS28)-CRP (http://www. das-score.nl/) higher than 2.6 irrespective of taking disease modifying anti-rheumatic drugs and/or corticosteroid. In patients positive for amyloid deposition on histological examinations we reevaluated magnetic resonance imaging (MRI) of the knee joint taken before surgery in order to find abnormal findings suggestive of this pathology [8-10].

\section{Histopathological study}

Five $\mu \mathrm{m}$-thick sections prepared from formalin-fixed specimens were put on the glass slides, and were processed for phenol Congo red staining as well as immunohistochemistry. Phenol Congo red staining has been described elsewhere [11]. The materials were examined under a polarizing microscope (Nikon, Eclipse, E600 POL) and the presence of amyloid was confirmed by its characteristic apple-green birefringence. Immunohistochemical staining was carried out using a Ventana XT automated immunohistochemistry system (Ventana Medical Systems, Tucson, AZ) and the primary antibodies used were anti-A $\lambda$, anti-A $\kappa$, anti-AA, and anti-TTR [12]. For TTR immunostaining, all sections were pretreated with $98 \%$ formic acid for $5 \mathrm{~min}$.

$\underline{\text { DNA analysis }}$ 
If staining for TTR amyloid was positive, we extracted DNA from paraffin-embedded tissue sections on slide glasses using a commercially available kit (TaKaRa DEXPAT, Takara Bio, Otsu, Japan), and performed direct DNA sequencing of all 4 exons of the TTR gene in order to detect its mutations.

\section{$\underline{\text { Statistical analysis }}$}

To detect a significant difference in age of the patients between with and without amyloid deposition, we used the Mann-Whitney U test. Relationships between amyloid deposition and age or gender of the patients were investigated using the logistic regression test. The results were expressed as mean \pm standard deviation, and a p-value less than 0.05 was regarded as statistically significant. Commercially available statistics software was used for data analysis (StatView for Macintosh, Abacus Concepts, Berkeley, CA, USA).

\section{Results}

No patients met our exclusion criteria, and 322 specimens obtained from 232 patients were analyzed in this study. Ninety patients provided us with the synovial tissue from both sides of knee joints. The study population consisted of 31 men and 201 women ranging in age from 50 to 92 years (mean, $73.0 \pm 9.1$ years). Twenty-six specimens $(8.1 \%)$ obtained from 21 patients showed deposition of amyloid, which was positively stained with the anti-TTR antibody alone. Five patients showed amyloid deposition in the synovial tissue of both knee joints. The TTR amyloid-positive patients consisted of 5 men and 16 women ranging in age from 71 to 89 , and their clinical diagnoses were OA alone in 19 and OA with inactive RA in 2. Both patients with OA with inactive RA had treatment histories of the latter using methotrexate for longer than 2 years, and had persistently shown DAS28-CRP lower than 2.6 for at least 1 year before surgery. There was a significant difference in age of the patients between with (mean, $79.0 \pm 4.6$ years) and without TTR amyloid deposition (mean, $72.4 \pm 9.3$ 
years) $(\mathrm{p}<0.001)$. Prevalence of TTR deposition in the patients separated by age and gender is demonstrated in Figure 1. The logistic regression test showed that aging (odds ratio, 71.02; 95\% confidence interval, 4.25-1831.08) is an independent risk factor for TTR amyloid deposition in knee joints. According to localization TTR amyloid in the synovial tissue showed either of the following 2 deposition patterns: one was inhomogeneous accumulations in the loose connective tissue under the synovial epithelia (Figs.2A and B) sometimes with nodule formation (Figs. 2C and D) and the other was small vessel involvement in the adipose tissue (Figs. 2E and F). The former pattern was seen in 18 patients, in which 8 had nodule formation, while the remaining 3 showed the latter. Medical records of the TTR amyloid-positive patients revealed nothing remarkable in the clinical course, macroscopic intraarticular findings at surgery, ECG or chest X-ray. MRI images taken before surgery were reevaluated by 3 of the authors (TT, MM and HY), but no abnormal findings suggestive of amyloid deposition were detectable. We could not find any mutations in the TTR gene of the patients with amyloid deposition on the direct DNA sequencing method.

\section{Discussion}

A pathological study of knee joints in autopsy cases has demonstrated that deposition of amyloid can occur in the intraarticular structures, and among them the meniscus is most frequently involved [13]. According to this report and one other, 17 to $50 \%$ of the joint capsule, including the synovial tissue, also shows amyloid deposition $[13,14]$. In the present study, however, approximately $8 \%$ of the examined specimens showed deposition of amyloid, which was immunohistochemically identified as being of TTR origin. The discrepancy in the frequency of synovial amyloid deposition between the previous reports and present study may be chiefly due to the size of specimens. Focusing on the intraarticular synovial tissue alone in the present study may also have contributed to the low frequency of amyloid deposition. 
Other types of amyloid, such as AA, have been reported to accumulate in the synovial tissue, particularly in that of RA patients [14]. The present study included 2 patients who had undergone surgery for treatment of OA with inactive RA, but neither of them showed deposition of AA amyloid. DNA analysis revealed no pathogenic mutations in the gene of TTR, strongly suggesting that the wild type had turned into amyloid fibrils detected in the present study.

There are 2 important issues worth consideration: one is whether or not deposition of TTR amyloid found in the present study is a part of systemic amyloidosis. Kyle et al proposed a clinical entity designated as amyloidosis localized to the tenosinovium based on a retrospective study of 124 patients who had shown amyloid deposition at carpal tunnel release and good prognosis with no evidence of visceral organ involvement [15]. Amyloid fibrils in this disease have later been identified as being of TTR origin on immunohistochemistry [16]. Several recent reports have demonstrated that wild-type TTR-derived amyloid can accumulate in various ligaments and tendons, including flexor muscles at the wrists, and the vertebral bone irrespective of visceral organ involvement $[4,5,17]$. The precise reason why wild-type TTR-derived amyloid preferentially accumulates in these musculoskeletal tissues remains unclear, but long-term mechanical stress, such as excessive load and heavy motion, may be relevant to the pathogenesis [4]. Amyloid deposition in the superficial layer under synovial epithelia as seen in the present study also supports this hypothesis. Deformities with limited range of motion in joints ascribed to OA may have produced additional accelerating effects on amyloid deposition via increasing the mechanical stress, although there is no clear evidence in the present study. Wild-type TTR monomers probably underwent conformational changes in the synovial tissue of knee joints, resulting in the formation of amyloid fibrils in the loose connective tissue. Small vessel wall involvement in the adipose tissue as seen in the present study may also be due to amyloidosis localized to the tenosynovium, but another possibility is SSA, which often 
shows a similar pattern of amyloid deposition [2, 18, 19]. Amyloid deposition in the loose connective tissue was not concurrent with that in small vessel walls, suggesting that their pathogenetic mechanisms may be different from each other. Advanced age of the 70s or older in all of the patients with the small vessel wall deposition also supports a possible role of SSA in the development of this pathology, although there were no obvious clinical symptoms or laboratory data suggestive of cardiac involvement.

The other important issue worth consideration is whether or not wild-type TTR-derived amyloid in the synovial tissue plays a role in worsening of OA. A recent pathological study on a savannah monkey has demonstrated severe hemorrhage in the knee joints ascribable to massive deposition of wild-type TTR amyloid in the synovial tissue, in which many vessels are present [6]. Considering that involvement of small vessel walls was seen in the present study, friability of vascular walls may cause intraarticular hemorrhage, leading to rapid worsening of OA. In the present study, however, we could not find any obvious differences in either macroscopic intraarticular findings at surgery or the clinical course of OA between with and without amyloid deposition.

To detect amyloid deposition around joints MRI is practically useful [8, 20, 21]. Bone erosions in RA and some malignancies, such as multiple myeloma, usually show low- and high-intensity signals on T1- and T2-weighted images of MRI, respectively, while amyloid deposits, including those in the soft tissue, appear as low-intensity signals on both types of image [8-10, 22]. In the present study, however, we could not find any abnormal signals around the synovial tissue suggestive of amyloid deposition even on reevaluation of MRI after histological examinations. This is probably due to the small amount of deposited amyloid in the synovial tissue, which was detectable only by microscopic examination. Bone scintigraphy with a high affinity to amyloid fibrils, particularly TTR-derived ones, is another possible way to detect minute deposition of amyloid as seen in the present study [23, 24], but 
distinction from other pathologies, such as inflammation, may be difficult.

In conclusion, deposition of wild-type TTR-derived amyloid can occur in the synovial tissue of the knee joints probably as a result of mechanical stress and/or as a manifestation of SSA. Nevertheless, whether or not this type of amyloidosis can accelerate OA remains unclear. Further studies in a larger set of patients are necessary in order to clarify the clinical significance of amyloid deposition in the intraarticular synovial tissue.

\section{Acknowledgment}

We appreciate Dr. Y. Fujinaga for his advice and suggestions in reevaluating MRI images of the knee joint. We are grateful to Ms. M. Nakajima for her help in statistical analyses and Ms. E. Nomura for her technical assistance in Congo red staining and immunohistochemistry. Informa Healthcare, the publisher of Amyloid, had the kindness to allow us to archive this article.

\section{Declaration of Interest}

This work was supported in part by a grant from the Intractable Disease Division, the Ministry of Health and Welfare, Amyloidosis Research Committee in Japan. 


\section{References}

1. Connors LH, Doros G, Sam F, Badiee A, Seldin DC, Skinner M. Clinical features and survival in senile systemic amyloidosis: comparison to familial transthyretin cardiomyopathy. Amyloid 2011; 18 (Suppl 1): 152-154.

2. Ikeda S. Senile systemic amyloidosis: clinical pictures and diagnostic criteria. Amyloid 2011; 18 (Suppl 1): 150-151.

3. Westermark P, Sletten K, Johansson B, Cornwell GG 3rd. Fibril in senile systemic amyloidosis is derived from normal transthyretin. Proc Natl Acad Sci USA 1990; 87: $2843-2845$

4. Sekijima Y, Uchiyama S, Tojo K, Sano K, Shimizu Y, Imaeda T, Hoshii Y, et al. High prevalence of wild-type transthyretin deposition in patients with idiopathic carpal tunnel syndrome: a common cause of carpal tunnel syndrome in the elderly. Hum Pathol 2011; 42: 1785-1791.

5. Sueyoshi T, Ueda M, Jono H, Irie H, Sei A, Ide J, Ando Y, et al. Wild-type transthyretin-derived amyloidosis in various ligaments and tendons. Hum Pathol 2011; 42: 1259-1264.

6. Chambers JK, Kanda T, Shirai A, Higuchi K, Ikeda S, Une Y. Senile systemic amyloidosis in an aged savannah monkey (Cercopithecus aethiops) with tenosynovial degeneration. J Vet Med Sci 2010; 72: 657-659.

7. Altman R, Asch E, Bloch D, Bole G, Borenstein D, Brandt K, Christy W, et al. Development of criteria for the classification and reporting of osteoarthritis: classification of osteoarthritis of the knee. Arthritis Rheum 1986; 29: 1039-1049.

8. Cobby MJ, Adler RS, Swartz R, Martel W. Dialysis-related amyloid arthropathy: MR findings in four patients. AJR Am J Roentgenol 1991; 157: 1023-1026.

9. Fautrel B, Fermand JF, Sibilia J, Nochy D, Rousselin B, Ravaud P. Amyloid arthropathy in the course of multiple myeloma. J Rheumatol 2002; 29: 1473-1481. 
10. Sheldon PJ, Forrester DM. Imaging of amyloid arthropathy. Semin Musculoskelet Radiol 2003; 7: 195-203.

11. Ishii W, Matsuda M, Nakamura A, Nakamura N, Suzuki A, Ikeda S. Abdominal fat aspiration biopsy and genotyping of serum amyloid A contribute to early diagnosis of reactive AA amyloidosis secondary to rheumatoid arthritis. Intern Med 2003; 42: 800-805.

12. Hoshii Y, Setoguchi M, Iwata T, Ueda J, Cui D, Kawano H, Gondo T, et al. Useful polyclonal antibodies against synthetic peptides corresponding to immunoglobulin light chain constant region for immunohistochemical detection of immunoglobulin light chain amyloidosis. Pathol Int 2001; 51: 264-270.

13. Ladefoged C. Amyloid deposits in the knee joint at autopsy. Ann Rheum Dis 1986; 45: 668-672.

14. Saitou H. Amyloid deposition in chronic joint disease. J Jpn Orthop Assoc 1994; 68: 534-544 (in Japanese with English abstract).

15. Kyle RA, Eilers SG, Linscheid RL, Gaffey TA. Amyloid localized to tenosynovium at carpal tunnel release. Natural history of 124 cases. Am J Clin Pathol 1989; 91: 393-397.

16. Kyle RA, Gertz MA, Linke RP. Amyloid localized to tenosynovium at carpal tunnel release. Immunohistochemical identification of amyloid type. Am J Clin Pathol 1992; 97: 250-253.

17. Sueyoshi T, Ueda M, Sei A, Misumi Y, Oshima T, Yamashita T, Obayashi K, et al. Spinal multifocal amyloidosis derived from wild-type transthyretin. Amyloid 2011; 18: $165-168$.

18. Westermark P, Bergström J, Solomon A, Murphy C, Sletten K. Transthyretin-derived senile systemic amyloidosis: clinicopathologic and structural considerations. Amyloid 2003; 10 (Suppl 1): 48-54.

19. Ikeda S, Sekijima Y, Tojo K, Koyama J. Diagnostic value of abdominal wall fat pad 
biopsy in senile systemic amyloidosis. Amyloid. 2011: 18: 211-215.

20. Gisserot O, Landais C, Cremades S, Terrier JP, Leyral G, Bernard P, de Jauréguiberry JP. Amyloid arthropathy and Waldenstrom macroglobulinemia. Joint Bone Spine 2006; 73: 456-458.

21. Katoh N, Tazawa K, Ishii W, Matsuda M, Ikeda S. Systemic AL amyloidosis mimicking rheumatoid arthritis. Intern Med 2008; 47: 1133-1138.

22. Baur-Melnyk A, Buhmann S, Dürr HR, Reiser M. Role of MRI for the diagnosis and prognosis of multiple myeloma. Eur J Radiol 2005; 55: 56-63.

23. Janssen S, Rijswijk MH, Piers DA, de jong GM. Soft-tissue uptake of ${ }^{99 m}$ Tc-diphosphonate in systemic AL amyloidosis. Eur J Nucl Med 1984; 9: 538-541.

24. Zwas ST, Shpilberg O, Huszar M, Rozenman J. Isolated ectopic lung uptake of technetium 99m methylene diphosphonate on bone scintigraphy in primary amyloidosis. Eur J Nucl Med 1990; 17: 282-285. 


\section{Figure legends}

Figure 1: Prevalence of TTR amyloid separated by age in all patients examined $(n=232)$, male patients $(n=31)$, and female patients $(n=201)$. Data are expressed as relative percentages, with the number of TTR amyloid-positive patients and number of patients tested in each age group indicated above each bar.

Figure 2: Histopathology of the synovial tissue obtained from 18 patients showed inhomogeneous deposition of amyloid in the superficial loose connective tissue under the epithelia (arrows in A, Congo red, bar=10 $\mu \mathrm{m}$ ) sometimes with nodular formation (C, Congo red, bar=10 $\mu \mathrm{m})$, which was immunoreactive to the anti-transthyretin antibody (B and $\mathrm{D}$, bars $=10 \mu \mathrm{m}$ ). In the remaining 3 patients amyloid deposition was seen in the wall of small vessels in the adipose tissue (E, Congo red, bar $=5 \mu \mathrm{m})$. These deposits were immunoreactive to the anti-transthyretin antibody $(\mathrm{F}$, bar $=5 \mu \mathrm{m})$. 
All

patients

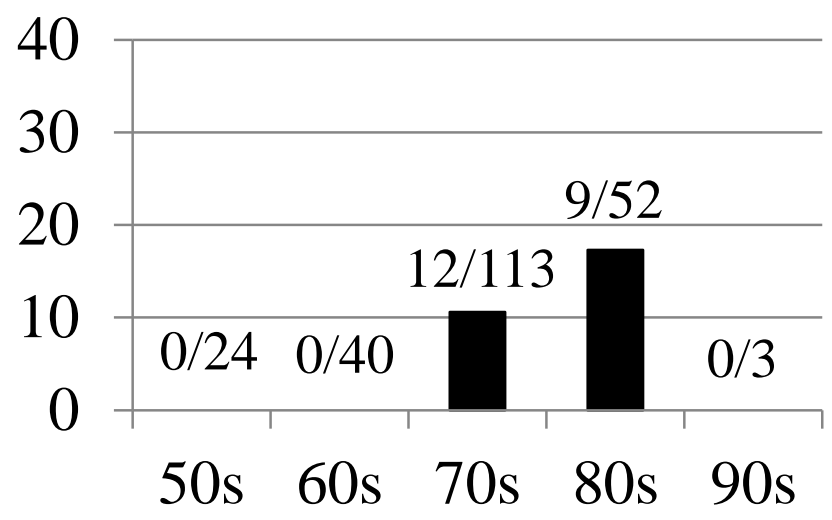

Male
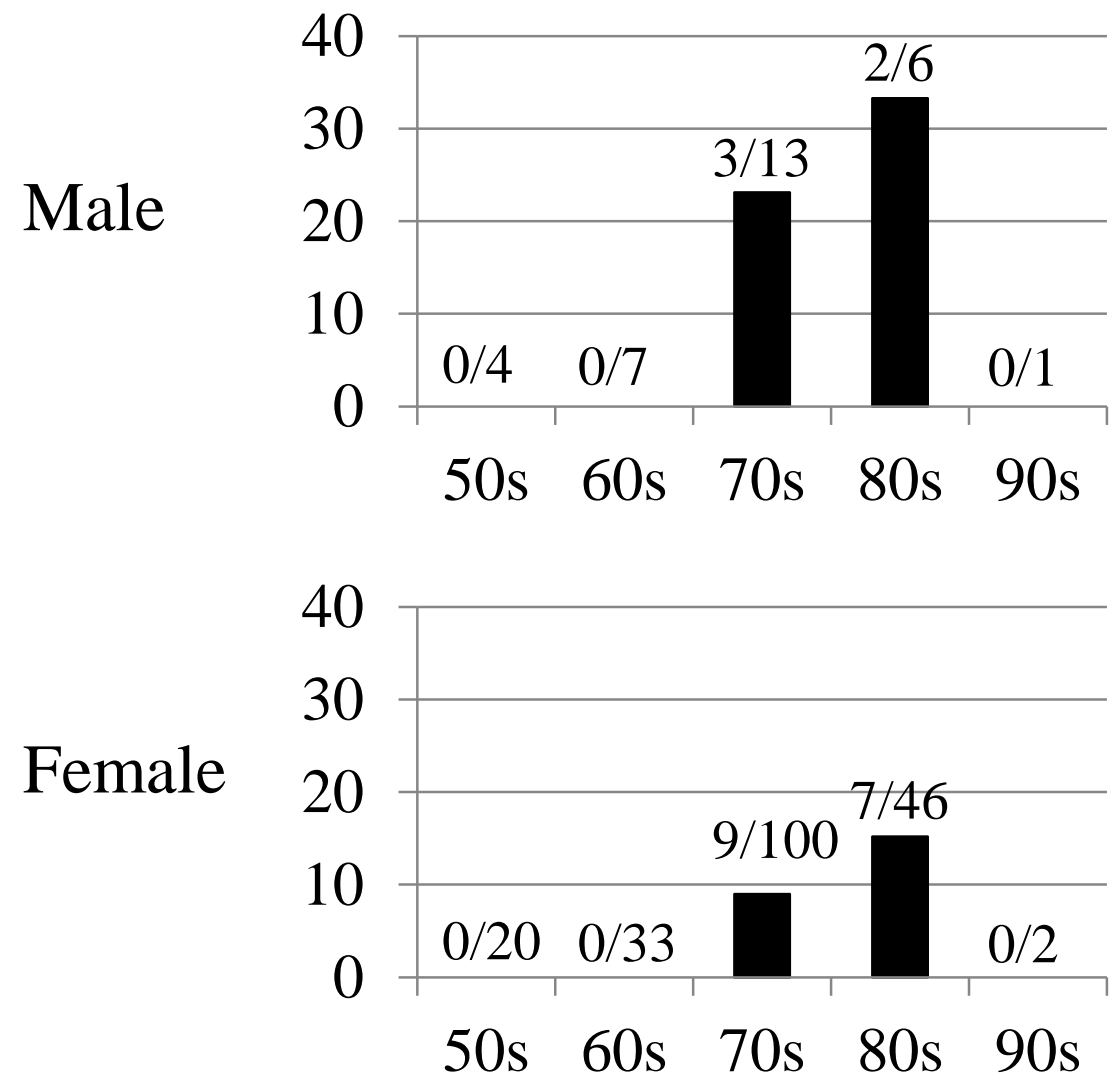

Figure 1 

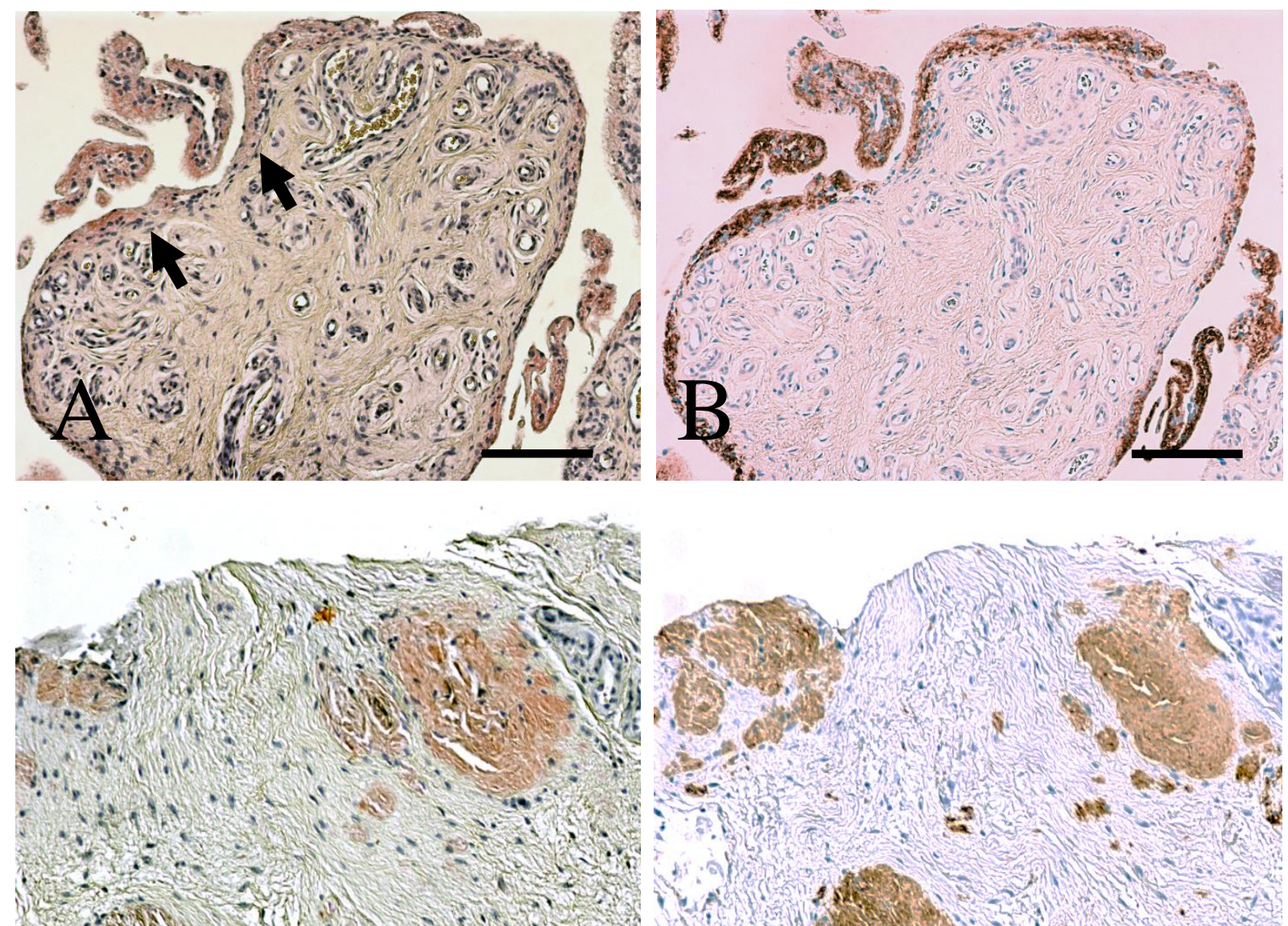

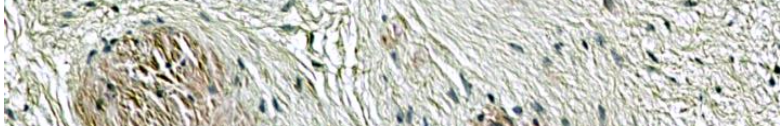
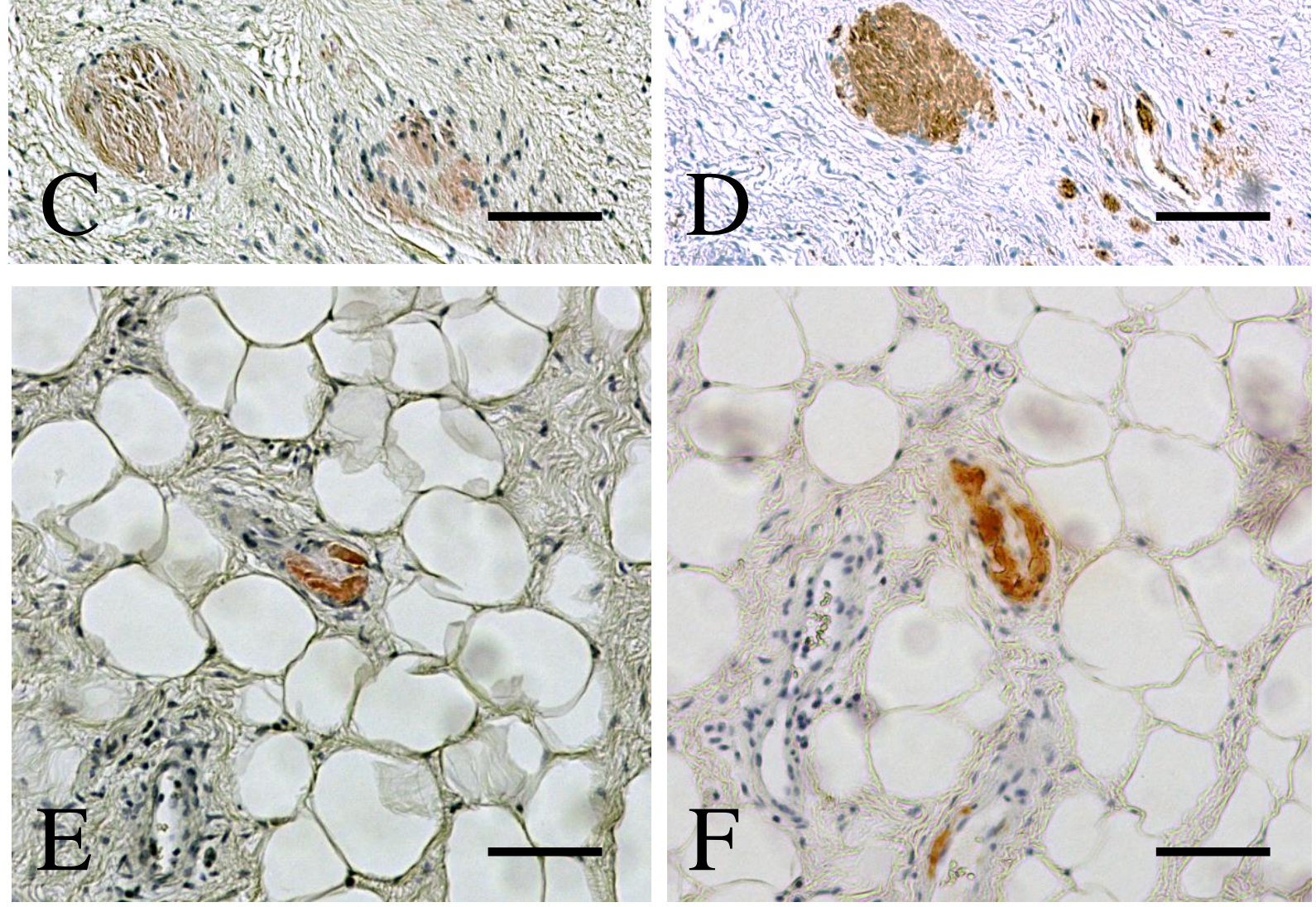